\title{
Proceedings of the 21st International Stroke Genetics Consortium Workshop
}

Neurol Genet 2018;4:e233. doi:10.1212/NXG.0000000000000233

\section{Introduction and welcome}

\author{
M. Fornage ${ }^{1}$ \\ ${ }^{1}$ Institute of Molecular Medicine and Human Genetics Center, the University of Texas Health Science Center at \\ Houston, TX.
}

The 21st workshop and 10th anniversary of the International Stroke Genetics Consortium (ISGC) was held on June 1-2, 2017 in Houston, TX, hosted by Myriam Fornage and Ynte Ruigrok. The ISGC is an international collaboration of physicians and scientists who have agreed to pool resources and expertise in an effort to unravel the genetic basis of stroke and its comorbidities (http://www.strokegenetics.org). It was formed in 2007 by a small group of scientists and has since grown to over 200 members representing over 50 countries in North and South America, Europe, Australia, and Africa. ISGC workshops are held semiannually and provide a forum for ISGC scientists and researchers to report on progress of ongoing scientific projects and discuss new ideas that help advance the ISGC's research mission. The ISGC nurtures its junior members offering up to 2 travel awards to young investigators. Many abstracts in these Proceedings reflect the contributions of active junior investigators. Herein, we present the Proceedings and official published abstracts of the 21 st ISGC workshop.

Presentations at the workshop included invited lectures, ISGC working group reports, and short presentations about ongoing research projects and new collaborative ideas or proposals. Major themes of the 21st ISGC workshop revolved around the development and implementation of cloud-based computing platforms to drive discoveries in cardiovascular disease and stroke; the analysis of large, multi-ethnic samples to identify novel stroke genes; and the integration of functional genomic approaches to human genetic studies to define the biological mechanisms underlying stroke risk. Notably, the NINDS-funded "Platform for Accelerating Genetic Discovery for Cerebrovascular Disease" represents an important tool developed by the ISGC to make its primary data and genetic analysis results readily available to the wide scientific community to enhance discoveries in stroke genetics.

On behalf of the ISGC Steering Committee and its many members, we hope that you enjoy reviewing these Proceedings and we welcome opportunities for new collaborations.

Acknowledgment: Steering Committee-Daniel Woo, MD; Scientific CommitteeChristopher D. Anderson, MD, MMSc; Outreach Committee-Jennifer Majersik, MD; Analysis Committee-Rainer Malik, PhD; Imaging Committee-Natalia Rost, MD, MSc; Translational Research Committee-Christopher D. Anderson, MD, MMSc.

Correspondence Dr. Fornage myriam.fornage@uth.tmc.edu 


\section{Intracranial aneurysm genetics group: Available infrastructure}

P. Bijlenga ${ }^{1}$, S. Morel ${ }^{1}$, T. Foroud ${ }^{2}$, J. Pera ${ }^{3}$, E. Gaal-Paavola ${ }^{4}$, M. Niemelä ${ }^{5}$, J. Broderick ${ }^{6}$, B. Worrall ${ }^{7}$, D. Woo ${ }^{6}$, and Y. Ruigrok ${ }^{8}$

${ }^{1}$ University of Geneva, Switzerland; ${ }^{2}$ Indiana University School of Medicine, Indianapolis; ${ }^{3}$ Jagiellonian University, Krakow, Poland; ${ }^{4}$ Kuopio University Hospital, Finland; ${ }^{5}$ University of Helsinki and Helsinki University Hospital, Finland; ${ }^{6}$ University of Cincinnati, $\mathrm{OH} ;{ }^{7}$ University of Virginia School of Medicine; and ${ }^{8}$ University Medical Center, Utrecht, The Netherlands.

Objective Exploring the genetics' associated with the formation, progression and rupture of intracranial aneurysms. Background Intracranial aneurysm is a multifactorial disease resulting from a disequilibrium between injury and repair. Genetics may contribute to better identify patients at risk, classify cases in disease subtypes, better understand the pathophysiology and identify potential therapeutic targets. Exploring the genetics of intracranial aneurysm requires a high volume of cases documented with high quality data covering the phenotype description and genotype. A multi-center international collaboration is necessary. Design/Methods An infrastructure to manage collaboration has been set up. A list of project has been prioritized. Data has been collected, harmonized, and tools to explore the data and generate optimal study groups to be compared developed. Results A standard data set including the basis of recruitment, gender, age at diagnosis, IA familial history status, smoking status, number of IA, IA location, size and rupture status of genotyped patients and healthy volunteers has been harmonized from 13 sources world-wide. GWAS data is available for 4,002 patients. Associated phenotypic data is available currently for 3,492 patients (2,346 females, 1,145 males), 1,106 patients with a positive familial history, 2,102 patients with sporadic IA. Smoking status is known for 3,742 subjects, 1,395 never smokers and 2,346 former or current smokers. Age at aneurysm rupture is known for 2,441 patients. Number of aneurysms is known for 3,372 patients, 956 with multiple aneurysms and 2,416 with a single aneurysm. Rupture status of aneurysm is known for 3,342 cases, 2,323 ruptured and 1,019 unruptured. Aneurysm location is known for 3,333 cases, 891 Acom aneurysm, 843 MCA aneurysms, 598 ICA aneurysms, 849 Pcom and vertebrobasilar aneurysms. Size of aneurysm at rupture is known in 1,461 patients, 782 are less than $7 \mathrm{~mm}, 537$ between 7 and $12 \mathrm{~mm}, 126$ between 12 and $25 \mathrm{~mm}, 15$ more than $25 \mathrm{~mm}$. Conclusions Efforts to collect and harmonize data from more sources is continuing. The working group has sufficient data to start subgroup analysis and is raising funds to further increase the numbers in the database and increase the statistical power.

Contact Email: philippe.bijlenga@hcuge.ch

Disclosure and Study Support: No financial interests or potential conflicts of interest.

\section{Genetic imbalance is associated with less favourable outcome after ischemic stroke}

A. Lindgren ${ }^{1}$, K. Schlicht ${ }^{2}$, D. Pfeiffer ${ }^{3}$, B. Chen ${ }^{4}$, S. Bevan ${ }^{5}$, S.Engelter ${ }^{6}$, C. Jern ${ }^{7}$, J.Jimenez-Conde ${ }^{8}$, J.-M. Lee ${ }^{9}$, R. Lemmens ${ }^{10}$, D. Leys ${ }^{11}$, B. Mitchell ${ }^{12}$, A. Pezzini ${ }^{13}$, J. Rosand ${ }^{14}$, N. Rost ${ }^{14}$, T. Tatlisumak, ${ }^{75}$, V. Thijs ${ }^{16}$, D. Woo ${ }^{17}$, S. Freitag-Wolf', M. Krawczak ${ }^{2}$, S. Debette ${ }^{18}$, S. Kittner ${ }^{12}$, J.W. Cole ${ }^{12}$, and C. Grond-Ginsbach ${ }^{3}$, On behalf of the Cervical Artery Dissections and Ischemic Stroke Patients (CADISP) and Stroke Genetics Network (SiGN) studies

${ }^{1}$ Department of Clinical Sciences, Neurology, Lund University, Sweden; ${ }^{2}$ Institute of Medical Informatics and Statistics, Kiel University, Germany; ${ }^{3}$ Department of Neurology, University of Heidelberg, Germany; ${ }^{4}$ Department of Biology, Southern University of Science and Technology, Shenzhen, China; ${ }^{5}$ School of Life Sciences, University of Lincoln, UK; ${ }^{6}$ Department of Neurology and Stroke Center, University Hospital Basel, Switzerland; ${ }^{7}$ Institute of Biomedicine, Sahlgrenska Academy, University of Gothenburg, Sweden; ${ }^{8}$ Department of Neurology, Institut Hospital del Mar d'Investigació Médica, Barcelona, Spain; ${ }^{9}$ Department of Neurology, Washington University School of Medicine, St Louis; ${ }^{10}$ Department of Neurosciences, University of Leuven, Belgium; ${ }^{11}$ Department of Neurology, University of Lille, France; ${ }^{12}$ Department of Neurology University of Maryland School of Medicine, Baltimore; ${ }^{13}$ Department of Clinical and Experimental Sciences, Neurology Clinic, University of Brescia, Italy; ${ }^{14}$ Department of Neurology, Massachusetts General Hospital, Harvard Medical School, Boston; ${ }^{15}$ Department of Neurology, Helsinki University Hospital, Finland; ${ }^{16}$ Department of Neurology, Austin Health, Heidelberg, Australia; ${ }^{17}$ Department of Neurology and Rehabilitation, University of Cincinnati; and ${ }^{18}$ INSERM U1219 and Department of Neurology, University of Bordeaux, France.

Objective To examine if genetic imbalance is associated with functional outcome after ischemic stroke. Background Genetic imbalance occurs when a protein-coding gene has more or fewer copies than the 2 copies of a normal diploid genome. Such imbalance may affect protein levels or their proportion relative to other proteins in genes that otherwise may have normal sequence. Ohnologs are genes with pronounced dosesensitivity. Imbalance affecting more than 5 protein-coding genes can be classified as "large imbalance." We explored the impact of genetic imbalance on outcome after ischemic stroke (IS) in 2 independent study samples. Design/Methods Copy number variation was detected by PennCNV analysis of GWAS-microarrays. Findings were individually inspected after noise reduction (http://noise-free-cnv.sourceforge.net/). True findings were mapped on the human genome (http:// www.ensembl.org/index.html) and the ohnolog repository (http://ohnologs.curie.fr/). Genetic imbalance was studied 
in IS patients with favorable (mRS 0-2) and unfavorable (mRS 3-6) outcome after 3 months from the CADISP (Cervical artery dissection and ischemic stroke patients) study $(\mathrm{n}=816$; age $=44 \pm 10$ years $)$. To validate the findings, similar analyses were performed in IS patients from $6 \mathrm{SiGN}$ centers $(n=2,498$; age $=68 \pm 14$ years $)$. In the logistic regression analyses a propensity score was used to adjust for age, sex, stroke severity (NIHSS), stroke subtype (CCS or TOAST) and center of recruitment. Results In the CADISP sample, patients with large imbalance were less likely to have favorable outcome $(p=0.0013$; Odds Ratio $=0.20$; $95 \%$ Confidence Interval $=0.08-0.57)$. The association of large imbalance with unfavorable outcome was also observed in the SiGN sample $(p=0.021$; OR $=0.61 ; 95 \% \mathrm{CI}=0.40-0.93)$. Genetic imbalance was also analyzed as a continuous variable by counting the number of imbalanced protein-coding genes per patient. The number of imbalanced genes was associated with unfavorable outcome in CADISP $(p=0.0042$; OR = $0.91 ; 95 \% \mathrm{CI}=0.85-0.98)$ as well as in SiGN ( $p=0.0064$; OR $=0.95 ; 95 \% \mathrm{CI}=0.92-0.99$ ). Imbalance affecting an ohnolog was associated with unfavorable outcome in the SiGN sample $(p=0.020 ; \mathrm{OR}=0.66 ; 95 \% \mathrm{CI}=0.47-0.94)$ and showed a trend towards association in the CADISP sample $(p=0.101$; $\mathrm{OR}=0.45 ; 95 \% \mathrm{CI}=0.18-1.23)$. Conclusions Genetic imbalance is an independent predictor of unfavorable outcome three months after stroke.

Contact Email: arne.lindgren@med.lu.se

Disclosure and Study Support: No financial interests or potential conflicts of interest.

\section{Fetal-type posterior cerebral artery is not associated with increased risk of ischemic stroke in the ipsilateral posterior cerebral artery territory vs ipsilateral middle and anterior cerebral artery territories}

P. Frid ${ }^{1}$, J. Wasselius ${ }^{2}$, A.-K. Giese ${ }^{3}$, J.W. Cole ${ }^{4}$, P.F. McArdle, J.P. Broderick, J. Jimenez-Conde ${ }^{7}$, C. Jern ${ }^{8}$, B.M. Kissela ${ }^{6}$, S.J. Kittner $^{4}$, D.O. Kleindorfer ${ }^{6}$, R. Lemmens ${ }^{9}$, J.F. Meschia ${ }^{10}$, B.D. Mitchell $^{5}$, J. Rosand ${ }^{3}$, T. Rundek ${ }^{11}$, R.L. Sacco ${ }^{11}$, R. Schmidt ${ }^{12}$, P. Sharma ${ }^{13}$, A. Slowik ${ }^{14}$, V. Thijs ${ }^{15}$, D. Woo ${ }^{6}$, B.B. Worrall ${ }^{16}$, Ona $W u^{17}$, J. Petersson ${ }^{1}$, N.S. Rost ${ }^{3}$, and A. Lindgren ${ }^{1}$, On behalf of the SiGN and MRI-GENIE studie

${ }^{1}$ Department of Clinical Sciences Lund, Neurology, Lund University, Sweden; Department of Neurology and Rehabilitation Medicine, Neurology, Skåne University Hospital, Lund, Sweden; ${ }^{2}$ Department of Clinical Sciences Lund, Diagnostic Radiology, Lund University and Department of Neuroradiology, Skåne University Hospital, Lund, Sweden; ${ }^{3}$ Department of Neurology, Massachusetts General Hospital,
Harvard Medical School, Boston; ${ }^{5}$ Department of Medicine, University of Maryland School of Medicine, Baltimore; ${ }^{4}$ Department of Neurology, University of Maryland School of Medicine and Veterans Affairs Maryland Health Care System, Baltimore; ${ }^{6}$ Department of Neurology and Rehabilitation Medicine, University of Cincinnati College of Medicine, $\mathrm{OH}$; ${ }^{7}$ Department of Neurology, Neurovascular Research Group (NEUVAS), IMIM-Hospital del Mar (Institut Hospital del Mar d'Investigacions Mèdiques), Universitat Autonoma de Barcelona, Spain; ${ }^{8}$ Institute of Biomedicine, the Sahlgrenska Academy at University of Gothenburg, Sweden; ${ }^{9} \mathrm{KU}$ Leuven-University of Leuven, Department of Neurosciences, Experimental Neurology and Leuven Research Institute for Neuroscience and Disease (LIND), Belgium; ${ }^{10}$ Department of Neurology, Mayo Clinic, Jacksonville, FL; ${ }^{11}$ Department of Neurology, Miller School of Medicine, University of Miami, FL; ${ }^{12}$ Department of Neurology, Clinical Division of Neurogeriatrics, Medical University Graz, Austria; ${ }^{13}$ Institute of Cardiovascular Research, Royal Holloway University of London (ICR2UL), Egham, UK; Ashford and St Peter's Hospital, UK; ${ }^{14}$ Department of Neurology, Jagiellonian University Medical College, Krakow, Poland; ${ }^{15}$ Department of Neurology Austin Health, Melbourne Brain Center, Florey Institute of Neuroscience and Mental Health, Heidelberg, Australia; ${ }^{16}$ Departments of Neurology and Public Health Sciences, University of Virginia, Charlottesville; and ${ }^{17}$ Department of Radiology, Athinoula A. Martinos Center for Biomedical Imaging, Massachusetts General Hospital, Charlestown.

Objective As an exploratory study within the Genetics in Posterior Circulation Stroke project, we aimed to determine the prevalence of fetal-type posterior cerebral artery (FPCA) in patients with acute ischemic stroke and its association with ischemic stroke in the ipsilateral posterior cerebral artery (PCA) vascular territory vs ipsilateral middle cerebral artery (MCA) and anterior cerebral artery (ACA) vascular territories. Background FPCA is a normal anatomic variant with the posterior cerebral artery (PCA) arising as a branch from the anterior cerebral circulation. Its reported prevalence varies (11-46\%) and its potential role in the pathophysiology of ischemic stroke has not been fully elucidated. Design/ Methods We reviewed acute ischemic stroke cases from 7 sites within the Stroke in Genetics Network (SiGN) image database. There were 682 ischemic stroke cases with MRI (DWI and MRA) data. A senior neuroradiologist blinded to clinical data and initial radiology assessment assessed all MRI data. Only cases with verified acute ischemic lesion(s) on MRI-DWI sequences and with MRA-TOF of intracranial vessels available were included in the analysis. We then excluded cases with infratentorial ischemic lesions and/or ischemic lesions in more than one vascular territory. Among the remaining 501 patients, we examined if the presence of FPCA was related to the proportion of patients with stroke in the ipsilateral PCA vs ipsilateral MCA/ACA territories. Results Of the 682 cases with available MRAs and acute ischemic DWI lesions, there were 59 (9\%) cases with unilateral, 
isolated PCA infarct(s). FPCA was observed in 205 cases (29\%): 144 (20\%) with unilateral FPCA and 61 (9\%) bilateral FPCA. The proportion of ischemic stroke did not differ in the presence of ipsilateral FPCA between the anterior (19\%) and PCA territory $(19 \%)(p=0.986)$ (Table 1). Conclusions In this study, a fetal-type posterior cerebral artery was not associated with an increased risk of ischemic stroke in the ipsilateral PCA territory compared to the ipsilateral MCA or ACA territories. Genetic variations influencing the presence of FPCA may therefore not be directly related to ischemic stroke location.

Optional upload of one figure, image or table:

\begin{tabular}{|r|r|r|r|}
\hline Ipsilateral FPCA & PCA Lesion & MCA/ACA Lesion & Total \\
\hline No, n (\%) & $48(81)$ & $360(81)$ & $408(81)$ \\
\hline Yes, n (\%) & $11(19)$ & $82(19)$ & $93(19)$ \\
\hline Total & $59(100)$ & 442 & 501 \\
\hline
\end{tabular}

Contact Email: arne.lindgren@med.lu.se

Disclosure and Study Support: No financial interests or potential conflicts of interest.

\section{Whole exome sequencing of young patients with familial aggregation of stroke in Lund Stroke Register}

A. Ilinca ${ }^{1}$, S. Samuelsson ${ }^{2}$, P. Piccinelli ${ }^{2}$, M. Soller ${ }^{2}$, U. Kristoffersson ${ }^{2}$, and A. Lindgren ${ }^{1}$

${ }^{1}$ Lund University, Skåne University Hospital; Department of Clinical Sciences Lund, Neurology, Sweden; and ${ }^{2}$ Department of Clinical Genetics, Lund University, Regional Laboratories, Region Skåne, Sweden.

Objective To use whole exome sequencing (WES) for detecting monogenic causes of ischemic stroke. Background We previously reported that familial clustering occurs in 13\% of young-onset stroke probands in Lund Stroke Register (LSR). From 4,467 probands, 20 probands aged under 55 years with familial aggregation of stroke were selected for WES to evaluate if possible monogenetic variations related to ischemic stroke could be detected. Design/Methods WES was performed with an amplicon-based library using Ion AmpliSeq Exome RDY panel. Based on Online Mendelian Inheritance in Man and reports in the literature, we compiled a list of 126 genes known or suspected to cause monogenic ischemic stroke, of which 11 are in the mitochondrial genome. We filtered the WES results for variants within the exons of the 115 non-mitochondrial genes. Variants with a minor allele frequency of $>1 \%$ in publically available databases were eliminated. The clinical phenotypes of the proband and affected family members were compared with clinical characteristics associated with reported pathogenic variants in the 115 genes, and mutation location (intronic/exonic/splice variants), type (synonymous, missense, frameshift) and possible previous reports in clinically similar diseases were evaluated. Results A mean of 19 variants in the 115 genes (range 11-26) per proband were identified. For 2 probands, we detected a previously reported pathogenic mutation. A previously reported pathogenic mutation/possible pathogenic mutation were detected for 9 of 20 probands. A mean of 2 mutations (0-4) per proband were compatible with the individual proband's and family's clinical stroke type and had possibly deleterious effect. Conclusions WES of young stroke patients with positive family history may reveal a monogenic cause of the disease. Co-segregation of the identified novel variants with stroke in the selected families will now be analyzed.

Contact Email: arne.lindgren@med.lu.se

Disclosure and Study Support: No financial interests or potential conflicts of interest.

\section{Interrogating stroke-associated loci using a Bayesian multinomial approach in $>20,000$ stroke cases and $>35,000$ controls}

\author{
M. Traylor ${ }^{1}$, and R. Malik ${ }^{2}$, On behalf of the Stroke Genetics \\ Network (NINDS-SIGN), UK Young Lacunar DNA Resource, \\ ISGC ICH GWAS Study Collaboration
}

${ }^{1}$ Stroke Research Group, University of Cambridge, UK; and
${ }^{2}$ Ludwig-Maximillians-Universität, Munich, Germany.

Objective To identify the causal variants and stroke phenotypes affected by genetic variation underlying loci associated with stroke. Background Large-scale genome-wide association studies have succeeded in identifying many loci associated with stroke phenotypes. The challenge now lies in interpreting how these variants confer risk of disease, and which stroke phenotypes are affected. A multinomial approach, which models all stroke phenotypes simultaneously against a set of shared controls, may aid characterization of such loci. Design/Methods We analyzed loci previously associated with stroke using a Bayesian Multinomial approach in $>20,000$ cases and $>35,000$ controls from the Stroke Genetics Network ( $\mathrm{SiGN}$ ), UK Young Lacunar stroke database, ISGC ICH GWAS Study collaboration, and other available cohorts, all of which were imputed to the Haplotype Reference Consortium. The multinomial approach enabled us to model all stroke phenotypes simultaneously; cardioembolic, large artery atherosclerosis, small vessel stroke, undetermined stroke, and intracerebral hemorrhage, against a shared set of 
controls. We used Akaike information criterion (AIC) and Bayesian information criterion (BIC) to evaluate whether multiple SNPs contribute to the signal at each locus and evaluated the set of stroke phenotypes most likely to explain the association at each locus using Bayesian model selection. Results We confirmed the existence of multiple independent signals at the PITX2 locus and found evidence suggesting that several other loci harbor multiple stroke-associated variants. Some loci, such as PITX2 and HDAC9, are specific to single stroke phenotypes, whereas others appear to confer risk across all, or multiple, stroke subtypes. Conclusions A multinomial logistic regression approach has value for interrogating stroke loci, and can provide novel information on affected phenotypes and potential causal variants.

Contact Email: mt628@medschl.cam.ac.uk

Disclosure and Study Support: Study Supported By: British Heart Foundation (BHF), Wellcome Trust, Stroke Association, $\mathrm{NIH} /$ National Institute of Neurological Disorders and Stroke U01 NS069208, NINDS R01 NS059727 and NINDS R01 NS036695. Disclosures: None.

\section{Genome wide study for rare variants reveals loci associated with hemorrhagic transformation after rT-PA}

C. Carrera ${ }^{1}$, N. Cullell ${ }^{2}$, N. Torres ${ }^{2}$, E. Muiño Acuña ${ }^{3}$, J. JimenezConde $^{3}$, J. Roquer ${ }^{3}$, A. Dávalos ${ }^{3}$, J. Arenillas $^{1}$, C. Vives-Bauza ${ }^{1}$, L. Heitsch ${ }^{5}$, L. Ibanez ${ }^{5}$, D. Strbian ${ }^{4}$, T. Tatlisumak, C. Cruchaga ${ }^{5}$, J. Montaner ${ }^{1}$, J.M. Lee $e^{5}$ and I. Fernández $z^{2}$

${ }^{1}$ Vall d'Hebron Institute of Research, Barcelona, Spain; ${ }^{2}$ Fundació Docència i Recerca Mutua Terrassa, Spain; ${ }^{3}$ IMIM-Hospital del Mar, Barcelona, Spain; ${ }^{4}$ Helsinki University Hospital, University of Helsinki, Finland; and ${ }^{5}$ Department of Neurology, Division of Emergency Medicine and Department of Psychiatry, Saint Louis, MO.

Objective We aimed to identify low-frequency genetic variants associated with Hemorrhagic Transformation (HT) after t-PA administration. Background Hemorrhagic transformation (HT) is a potentially fatal complication after t-PA treatment of ischemic stroke patients and appears to be genetically modulated. Low-frequency genetic variants could contribute in the genetic risk to complex traits including pharmacogenetics. Design/Methods We analyzed 1,195 t-PA treated ischemic strokes from Spain and Finland genotyped with Human Core Exome array (Illumina) using a gene-based approach. Minor allele frequency $<5 \%$ and nonsynonymous variants were selected. Quality controls and meta-analysis were performed through SKAT and MetaSKAT libraries from R and PLINK software. Sex, age, cardioembolic etiology and principal components were used as covariates under null model for global hemorrhagic transformation analysis. Results Two hundred thirteen participants developed HT based on ECASS criteria, 878 genes and 3,788 variants were analyzed. One gene was statistically associated with HT after Bonferroni correction $\left(p=5.4 \times 10^{-5}\right)$ and 14 genes presented a suggestive association $(p<0.001)$. The significant gene regulates action of RNA polymerase III and the synthesis of small RNAs. One of the suggestive genes was previously shown to be associated with inflammatory response and vascular homeostasis. Conclusions A gene-based approach of low frequency variants in t-PA treated patients has revealed a potential gene associated with HT following ischemic strokes. Replication and functional analysis will be needed to confirm these results.

\section{Contact Email: israelcadenas@yahoo.es}

Disclosure and Study Support: No disclosures. C-C. is supported by NIH R01 grant, GENISIS project. The Neurovascular Research Laboratory is supported by the Spanish stroke research network (INVICTUS plus), Pre-Test Stroke Project (PMP15/00022). Stroke Pharmacogenomics and Genetics Laboratory is supported by the Spanish stroke research network (INVICTUS plus), Generation Project (PI15/01978) and Pre-Test Stroke Project (PMP15/00022), Instituto de Salud Carlos III and Fondo Europeo de Desarrollo Regional (ISCIII-FEDER).

\section{Identifying and validating stroke diagnoses in UK Biobank}

K.N. Jun Hong ${ }^{1}$, A. Hutchison ${ }^{1}$, C. Schnier ${ }^{1}$, T. Wilkinson ${ }^{1}$, R. Woodfield ${ }^{1}$, Q. Zhang ${ }^{1}$, C.L.M. Sudlow ${ }^{1}$, and K. Rannikmäe ${ }^{1}$

${ }^{1}$ University of Edinburgh, UK Biobank.

Objective To assess the accuracy of stroke identification in UK Biobank. Background Stroke cases are identified in UK Biobank (UKB), a large population-based prospective study, through self-report at recruitment and linkage to routinelycollected, coded national health data. We assessed the accuracy of these sources in identifying stroke. Design/Methods We identified Edinburgh-based participants with stroke codes and extracted clinical information from their full text electronic hospital records. Specialist stroke physicians used this to generate a reference standard classification for each participant, confirming or rejecting the stroke diagnosis, and assigning a subtype diagnosis where possible. We calculated positive predictive values (PPVs) for strokes identified prior to recruitment ("prevalent") and after recruitment ("incident") through different sources (hospital admissions, primary care, or self-report). Results There were 275 participants with a code indicating a stroke; 161 had clinical information for adjudication, which was more comprehensive for hospital admitted cases. There were 71 prevalent strokes identified through hospital admissions (29), primary care data (28) or self-report alone (13). PPV for prevalent cases was $52 \%$ (95\% CI 41-63\%) overall but was higher for hospital 
admitted cases (72\%) than for primary care confirmed (50\%) or self-report only cases (15\%). There were 90 incident cases, of which 40 were first identified through hospital admissions and 50 through primary care. PPV for incident cases was 77\% (95\% 67-84\%), but was higher for hospital admitted (90\%) than for primary care confirmed cases (66\%). PPVs for incident stroke subtype cases identified through hospital admissions were $95 \%$ for ischaemic stroke $(n=21)$ and $67 \%$ for both intracerebral $(\mathrm{n}=3)$ and subarachnoid haemorrhage $(n=6)$. Conclusions Our results suggest that incident cases of stroke and ischaemic stroke are identified through linked hospital admissions data in UK Biobank with sufficient accuracy for genetic and epidemiologic research. Identification of haemorrhagic strokes appeared less accurate but numbers were small. PPVs for self-reported and primary care identified strokes may have been compromised by incomplete clinical information.

Contact Email: kristiina.rannikmae@gmail.com

Disclosure and Study Support: UK Biobank.

\section{Extracellular RNA-based biomarkers for neurological decline after aneurysmal subarachnoid hemorrhage}

\author{
M.Y.S. Kalani ${ }^{1}$, R.F. Spetzler ${ }^{2}$, M. Huntelman ${ }^{3}$, and K. Van \\ Keuren-Jensen ${ }^{3}$
}

${ }^{1}$ Department of Neurosurgery, University of Utah School of Medicine, Salt Lake City; ${ }^{2}$ The Barrow Neurologic Institute, Phoenix, AZ; and ${ }^{3}$ The Translational Genomics Research Institute, Phoenix, AZ.

Objective To develop a cerebrospinal fluid-based extracellular RNA panel predictive of neurologic decline after aneurysmal subarachnoid hemorrhage. Background Aneurysmal subarachnoid hemorrhage is associated with a $50 \%$ rate of death and in those who survive the ictus, a $50 \%$ rate of severe disability. Although the injury associated with the initial aneurysm rupture is difficult to mitigate, activation of secondary injury pathways after aneurysm rupture lead to further injury. Identification of markers predictive of the onset of neurologic decline will greatly aid practitioners in identifying at risk patients. Biomarkers predictive of activation of secondary injury pathways will also shed light on this uniquely human disease. Design/Methods All patients who presented with aneurysmal subarachnoid hemorrhage between 2013 and 2014 were enrolled in this study. Patients were enrolled within 24 hours of aneurysm rupture and were admitted to the intensive care unit where an external ventricular drain was placed and their aneurysm was secured within 24 hours of admission. Blood and cerebrospinal fluid was collected daily from patients from admission to the intensive care unit until the time of discontinuation of the external ventricular drain.
Additionally, patient clinical parameters pertinent to care of subarachnoid hemorrhage patients, including exams, intracranial pressure measurements, results of vascular imaging studies and transcranial Doppler, were collected prospectively. Extracellular RNA was harvested from blood and CSF and was subjected to RNA sequencing protocols. Results Using RNA sequencing algorithms, we identified a cohort of 143 differentially expressed microRNAs that predict cerebral vasospasm with an accuracy of $92 \%$ based on post-bleed day 2 cerebrospinal fluid. Furthermore, using a refined set of 7 microRNAs from this set we validated our finding. A cohort of 3 differentially expressed microRNAs can discern between those suffering from severe or mild vasospasm with an accuracy of $78 \%$. Conclusions Using patient samples and clinical information, we have identified extracellular RNAs that are predictive of neurologic decline after aneurysmal subarachnoid hemorrhage. Biomarkers predictive of neurologic decline are likely to help physicians stratify at risk patient populations and learn fundamental biology that is unique to this disease process.

Contact Email: yashar.kalani@hsc.utah.edu

Disclosure and Study Support: MYSK, MH and KVKJ are supported by NIH grant (1UH2TR000891/1UH3TR000891 \& Administrative Supplements).

\section{GWAS meta-analysis of stroke in individuals of African descent identifies 2 novel loci associated with stroke}

K.L. Keene ${ }^{1}$, H.I. Hyacinth ${ }^{2}$, B.B. Worrall ${ }^{3}$, and M. Fornage ${ }^{4}$, On behalf of the Consortium of Minority Population genome-wide Association Studies of Stroke (COMPASS)

${ }^{1}$ East Carolina University, Greenville, NC; ${ }^{2}$ Emory University, Atlanta, GA; ${ }^{3}$ University of Virginia School of Medicine, Charlottesville; and ${ }^{4}$ University of Texas Health Science Center at Houston.

Objective Stroke is a complex disease with multiple genetic and environmental risk factors. The objective of this study was to identify genetic contributors for stroke disparities in individuals of African descent. Background African Americans endure a nearly two-fold greater risk of having a stroke and are 2-3 times more likely to die from stroke compared to European Americans. Design/Methods The Consortium of Minority Population genome-wide Association Studies of Stroke (COMPASS) has conducted a comprehensive genome-wide association meta-analysis of stroke in more than 22,000 individuals of African ancestry (3,734 cases, 18,317 controls). Results Using sample size weighted and inverse variance weighted meta-analyses, we have identified three loci that reached genome-wide significance for association $(p<5 \times$ $\left.10^{-8}\right)$ and an additional 34 variants with suggestive evidence 
of association $\left(p<1 \times 10^{-6}\right)$, representing a total of 26 unique loci. For replication, a look-up analysis for a $100 \mathrm{~kb}$ region flanking the COMPASS SNP was performed in SiGN Europeans, SiGN Hispanics, and METASTROKE. Using a liberal Bonferroni correction $p$-value of $1.92 \times 10^{-3}$ for replication (0.05/26 unique loci), 2 of the three most significantly associated loci were replicated in one or more look-up analysis (SFXN4 and HNF1A). Conclusions Overall, 17 of 26 loci showed evidence for replication across multiple populations. Variants in the HNF1A gene have been previously associated with lipids, C-reactive protein, and risk of coronary artery disease and stroke. The SFXN4 gene represents a novel stroke locus and the encoded protein is critical for mitochondrial respiration and the production of red blood cells (erythropoiesis). Recent clinical trials suggest that erythropoiesisstimulating agents, while an effective treatment for anemia in patients with chronic kidney disease, also increases the risk of stroke possibly due to hyper-viscosity. In conclusion, these findings represent the most thorough investigation of genetic determinants of stroke in individuals of African descent, to date.

Contact Email: keenek@ecu.edu

Disclosure and Study Support: No financial interests or potential conflicts of interest.

\section{Stroke subtype phenotypes based on single items from the causative classification system (CCS) identify known genetic loci}

H. Ay ${ }^{1}$, B. Worrall ${ }^{2}$, P.McArdle ${ }^{3}$, J. Cole $^{3}$, J.Meschia $^{4}$, R. Brown ${ }^{5}$, K. Ryan ${ }^{3}$, R. Grewal ${ }^{6}$, S. Smoller ${ }^{7}$, C. Sudlow ${ }^{8}$, T. Rundek ${ }^{9}$, R. Sacco ${ }^{9}$, K. Rexrode ${ }^{1}$, A. Lindgren ${ }^{10}$, C. McDonough ${ }^{11}$, M. Ribases ${ }^{12}$, R. Zand ${ }^{13}$, V. Abedi ${ }^{13}$, B. Mitchell ${ }^{3}$, and S. Kittner ${ }^{3}$

${ }^{1}$ Harvard Boston; ${ }^{2}$ UVA, Charlottesville; ${ }^{3}$ UMSOM, Baltimore; ${ }^{4}$ Mayo, Jacksonville; ${ }^{5}$ Mayo, Rochester; ${ }^{6}$ Seton Hall University, NJ; ${ }^{7}$ Einstein NYC; ${ }^{8}$ University of Edinburgh; ${ }^{9} \mathrm{UM}$, Miami; ${ }^{10}$ Lund University; ${ }^{11} \mathrm{UF}$, Gainesville; ${ }^{12}$ VHIR, Barcelona; and ${ }^{13}$ Geisinger, Danville, PA.

Objective Compare the strength of association for known genetic signals between the established ischemic stroke (IS) subtyping systems (TOAST and the causative and phenotypic CCS subtypes) and phenotyping based on a single item within the CCS. Background Current IS subtyping systems are based on clinical reasoning, and are very labor-intensive and costly to implement at scale in large samples. There is a need to develop phenotyping approaches to be used in genetic studies that can be implemented using generally available items captured in electronic medical record systems. Design/Methods Non-mutually exclusive "Simple" IS subtypes were defined as follows: (1) Small vessel stroke-CCS item-2C-lacunar infarct on imaging (with no other imaging or other requirements); (2) large artery stroke-CCS item$3 \mathrm{~B}$ - stenosis in clinically relevant artery on vascular imaging judged to be due to atherosclerosis; and (3) cardioembolic stroke-CCS items-4b iii or -iv-persistent or paroxysmal atrial fibrillation. Using these definitions, the results of GWAS analysis among Europeans only were compared to the published results for TOAST and CCS. Results Compared to TOAST and the causative and phenotypic CCS subtypes, the "Simple" IS subtypes had similar strengths of association and $p$-values for the loci in the NINDS Stroke Genetic Network (SiGN) ischemic stroke GWAS study, Lancet Neurology 2015 PMID: 26708676 (see Table 1). Conclusions "Simple" IS subtypes perform as well in GWAS analyses as more complex phenotyping systems and could potentially be derived through automated or semi-automated methods applied to electronic medical record systems. Since the "simple" phenotypes were based on high quality CCS data, the current findings reflect optimal performance. Additional validation studies will be required for implementation in other settings.

Contact Email: skittner@som.umaryland.edu

Disclosure and Study Support: This work was supported by grant award number U01NS069208 from the National Institute of Neurological Disorders and Stroke (NINDS). There are no other disclosures or conflicts of interest.

\section{Association of heterozygosity for the sickle cell variant with stroke and cardiovascular disease in African Americans}
H.I. Hyacinth ${ }^{1}$, C. Carty ${ }^{2}$, S.R. Seals ${ }^{3}$, M.R. Irvin ${ }^{4}$, R.P. Naik ${ }^{5}$, M.C. Caughey, and N.A. Zakai ${ }^{7}$, On behalf of the sickle cell trait working group

${ }^{1}$ Emory University and Children's Health care of Atlanta, GA; ${ }^{2}$ University of Washington, Seattle; ${ }^{3}$ University of West Florida, Pensacola; ${ }^{4}$ University of Alabama at Birmingham; ${ }^{5}$ The Johns Hopkins University, Baltimore, MD; ${ }^{6}$ University of North Carolina, Chapel Hill; and ${ }^{7}$ University of Vermont, Burlington.

Objective The objective of this study was to determine whether individuals of African Ancestry who were heterozygous for the sickle cell mutation or carry the sickle cell trait (SCT), were at a significantly higher risk for incident ischemic stroke (IS), myocardial infarction (MI) or coronary heart disease (CHD) compared to those without SCT. Background Sickle cell trait (SCT), is the heterozygous carrier status of sickle cell disease in which the individual has one normal and one sickle hemoglobin gene. SCT trait has been associated with a hypercoagulable state, renal papillary necrosis, renal medullary carcinoma, and more recently kidney disease. The role of SCT as a risk factor for ischemic stroke and/or cardiovascular diseases has not been clearly resolved. Design/Methods This is a meta-analysis of 
genotype and phenotype data from the Women's Health Initiative (WHI) REasons for Geographic and Racial Differences in Stroke (REGARDS), Multi-Ethnic Study of Atherosclerosis (MESA), Jackson Heart Study (JHS) and Atherosclerosis Risk In Communities (ARIC) cohorts, ARIC. Due to overlap, ARIC could not contribute data to the meta-analysis for stroke. The outcomes were IS, MI or CHD. Incident IS was defined based on expert adjudication, MI was defined as adjudicated non-fatal or fatal $\mathrm{MI}$ and $\mathrm{CHD}$ was defined as (1) adjudicated non-fatal MI, (2) fatal MI, (3) documented coronary revascularization procedures or (4) non-MI CHD death. SCT status was determined by either direct genotyping or imputation for rs334 using the 1000 Genome reference panel. Homozygous individuals and those with a prior history of stroke or other cardiovascular diseases were excluded. Analysis was performed separately in each cohort using a Cox proportional hazard models to estimate the hazard ratio (HR) comparing SCT carriers to non-carriers. Models in each cohort were adjusted for age, sex, population stratification and other relevant covariates based on the Framingham stroke and CHD risk scores. Results from each cohort were then meta-analyzed using a random effect model. Results A total of 20,053 African American men and women were included in the combined sample; 1,503 with SCT (7.5\% prevalence). The HR for incident ischemic stroke was $0.58(0.14-2.14)$ in JHS, $0.65(0.15-2.73)$ in MESA, 0.69 (0.28-1.69) in REGARDS and 1.09 (0.47-2.51) in WHI; for MI was: $0.96(0.49-1.89)$ in WHI; $1.27(0.80-2.00)$ in REGARDS; 1.84 (0.74-4.60) in MESA; $1.24(0.28-5.44)$ in JHS; and $0.68(0.42-1.10)$ in ARIC for CHD it was: 1.05 (0.63-1.74) in WHI; 1.49 (1.01-2.18) in REGARDS; 2.82 (1.48-5.38) in MESA; 1.45 (0.50-4.19) in JHS; and 1.10 (0.80-1.50) in ARIC. Meta-analysis showed that, while SCT status was not significantly associated with incident ischemic stroke $(0.80[0.47-1.35])$ or MI (1.10 [0.73-1.64]), it was significantly associated with incident CHD (1.42 [1.02-1.98]). Conclusions Our results from the largest study of the association of SCT with stroke or cardiovascular disease in African Americans to date suggest that SCT is an independent risk factor for incident CHD but IS or MI in African Americans.

Contact Email: hhyacinth@emory.edu

Disclosure and Study Support: All authors have no relevant conflict of interest to disclose.

\section{SwissNeuroFoundation} and AneurysmDataBank

\section{P. Bijlenga ${ }^{1}$}

${ }^{1}$ Department of Neurosurgery, Hôpitaux Universitaires de Genève, Switzerland.

Intracranial aneurysms affect a significant proportion of the population. Due to advances in screening and cerebrovascular imagining, the diagnosis is shifted from symptomatic patients to asymptomatic individuals and often is an incidental finding. Once diagnosed, half the people incidentally diagnosed are offered interventions to secure the aneurysm and the others are prospectively observed with regular imaging. Less than $3 \%$ of patients regularly investigated cross from the observation cohort to intervention. Overall, probabilities suffering of: (1) an aneurysm (3\%), (3) aneurysm growth or new symptoms $(3 \% / y),(4)$ rupture $(1 \% / y)$ are small. When excluding the outcome associated with aneurysm rupture, complication resulting in ineffective treatment, morbidity and mortality of interventions is significant but relatively rare.

Therefore, most centers are confronted to small yearly caseloads (typically 100 to 200 cases/y) and despite the major impact of complications on patients and costs, the frequency is low ( $1 \%$ mortality and $5 \%$ morbidity). Concomitantly, clinicians are confronted to continuous developments of new decision models, treatment techniques and devices. Due to the low case-loads and the good but still unacceptably high risks, the evaluation of benefits associated with innovation requires multicenter and interdisciplinary collaboration. Evaluation of each case requires the balance of a minimum 3 factors (aneurysm location, size, intervention risk) and most probably many more i.e., genetics, vascular morphology, cerebro-vascular factors like blood pressure, flow, and viscosity. The understanding of the context associated with the management of each lesion requires the capture of multiple and heterogeneous descriptors. Individual regularly investigated and treated patients remain at risk all their life. The evaluation of the performance and quality of the disease management requires therefore a quantification of health using different tools and integrated over life. The main issue is to collect quickly sufficient good quality data to achieve sufficient statistical power to generate evidence.

The SwissNeuroFoundation (SNF) aims are to provide the community the infrastructure and tools to facilitate multicenter collaboration, harmonization of information collection, exploitation of data and dissemination of knowledge. The purpose is to improve the management of patients suffering of cerebro-vascular diseases and in particular intracranial aneurysms. The SNF develops a disease model and a Data Repository (AneurysmDataBank).

Disclosure and Study Support: No financial interests or potential conflicts of interest.

\section{Searching for biomarkers of prognosis in the acute phase of ischemic stroke}
A. Donatti ${ }^{1}$, R. Secolin ${ }^{1}$, S. Avancini ${ }^{1}$, B. Carvalho ${ }^{3}, W$. Avelar $^{2}$, and I. Lopes-Cendes ${ }^{1}$

${ }^{1}$ Departement of Medical Genetics; ${ }^{2}$ Departmanet of Neurology, School of Medical Sciences; and ${ }^{3}$ Department of 
Statistics, Institute of Mathematics, Statistics and Scientific Computing, University of Campinas-UNICAMP; and the Brazilian Institute of Neuroscience and Neurotechnology (BRAINN), Campinas, SP, Brazil.

Objective This is an ongoing project aiming to identify circulating biomarkers (microRNA and proteins) which could be used to determine prognosis and response to treatment in the acute phase of ischemic stroke. Background Recently, there has been an increasing interest in the identification of noninvasive circulating biomarkers, such as microRNAs and proteins which could be used to determine prognosis and response to different treatment. Design/Methods We are collecting samples at 2 distinct periods from the same patient: whiting the first 24 hours of the acute event and after 6 months. Plasma is separated from the leucocytes in a refrigerated centrifuge and storage at $-80^{\circ} \mathrm{C}$. MicroRNA extraction is performed mirVanaTM ParisTM kit (Thermo Fisher Scientific Inc). Small RNA sequencing will be performed using HiSeq 2500 (Illumina, Inc). In addition, plasma samples are been prepared for future proteomics studies. A number of clinical variables are also being collected including: demographics, risk factors, TOAST, Outcome scores (delta NIH, GENESIS project), treatment/ medication. In addition, CT scans and MRI are obtained for all patients. This project has been developed with the support of the acute endophenotype working group of the ISGC. Results To date, we have collected samples from 40 patients in both time-points. Our aim is to reach at least 100 patients, as has been indicated by power calculation as the minimum sample size required. We expect to have preliminary results from both microRNA-seq and proteomics soon. Conclusions As this is an ongoing project we are seeking collaboration from additional centers in order to reach the number of patients required.

Contact Email: icendes@unicamp.br

Disclosure and Study Support: This study is supported by grants from FAPESP (Fundação de Amparo a Pesquisa do Estado de São Paulo BRAZIL).

\section{Genome-wide association studies from the Vitamin Intervention for Stroke Prevention (VISP) trial detect novel loci for recurrent stroke}

K.L.Keene ${ }^{1}$, W.M.Chen ${ }^{2}$, F.C. Hsu ${ }^{3}$, S.R. Williams ${ }^{2}$, M.M. Sale ${ }^{2}$, and B.B. Worrall ${ }^{2}$, On behalf of the GARNET Collaborative Research Group

${ }^{1}$ East Carolina University, Greenville, NC; ${ }^{2}$ University of Virginia School of Medicine, Charlottesville, ${ }^{3}$ Wake Forest University School of Medicine, Winston Salem, NC.

Objective Our objective is to evaluate genetic contributors to recurrent stroke, a phenotype severely understudied.
Background Recurrent strokes account for $\sim 25 \%$ of all strokes annually, and are more-deadly and most likely to cause disability when compared to a first stroke. Design/Methods Our group has completed a genome-wide association study, identifying 2 novel loci associated with recurrent stroke in the Vitamin Intervention for Stroke Prevention (VISP) clinical trial population. A subset of 2,100 VISP participants were genotyped on the Illumina HumanOmnil-Quad BeadChip and survival analyses, in which days of follow-up to recurrent stroke was regressed on gender, age, treatment group, and principal components 1-4, were performed. Results These analyses detected 2 genome-wide significant associations (rs6664786; Beta $=-0.62, p=1.73 \times 10^{-8}$ and rs2184006; Beta $=-0.62, p=3.43 \times 10^{-8}$ ) located in a gene sparse region of chromosome 1 . Three additional SNPs in this region had nominal $p$-values ranging from $7.60 \times 10^{-7}$ to $8.23 \times 10^{-6}$. Subsequent treatment-stratified analyses of recurrent stroke, using Cox proportional hazards regression survival analysis, identified a cluster of nearly a dozen SNPs on chromosome 2 with genome-wide significant associations for recurrent stroke in the low dose treatment arm (adjusting for age, sex, and the top 5 principal components). The top SNP, rs6542775 (Beta $\left.=-1.966, p=2.19 \times 10^{-11}\right)$ is located downstream of the LIM and senescent cell antigen-like domains 1 (LIMS1) gene. Kaplan-Meier survival analyses by genotype for the top SNP, rs6542775 revealed that individuals in the low dose treatment arm carrying the GG genotype had significantly higher probability of longer survival (not suffering a recurrent stroke) than those with either the GA or AA genotypes ( $p=$ $\left.6.1 \times 10^{-8}\right)$. Conclusions Collectively, our findings suggest that variants across these 2 regions may have implications with regards to recurrent stroke risk and potential therapeutic response. Efforts are under way to perform a large scale metaanalysis for recurrent stroke.

Contact Email: keenek@ecu.edu

\section{Skeletal muscle DNA methylation changes in the lower extremity of chronic stroke patients}

H. Xu ${ }^{1}$, A.S. Ryan ${ }^{2}$, T.G. Forrester ${ }^{3}$, Y.C. Cheng ${ }^{1}$, A. Parihar ${ }^{1}$, K. Tanner ${ }^{1}$, J.W. Cole ${ }^{1,2}$, B.D. Mitchell ${ }^{1}$, C.E. Hafer-Macko ${ }^{1,2}$, and R.F. Macko ${ }^{1,2}$

${ }^{1}$ University of Maryland School of Medicine, Baltimore; ${ }^{2}$ Baltimore Department of Veterans Affairs Medical Center, $\mathrm{MD}$; and ${ }^{3}$ University of the West Indies, Mona Campus, Kingston, Jamaica.

Objective We hypothesize that hemiparetic stroke alters paretic leg skeletal muscle genomic DNA methylation profiles related to phenotypic abnormalities, compared to non-paretic leg. Background Stroke is the number one cause of serious long-term disability including extremity motor dysfunction in 
the United States. Skeletal muscle in paretic limbs switches toward fast twitch fiber-type with reduced oxidative capacity, increased intramuscular fat, inflammation, and atrophy, compared to non-paretic muscle. There are differences in muscle transcriptome profiles that relate to these specific phenotypic pathologies, which we postulate could result from post-stroke epigenetic changes. Design/Methods Five males (mean age $56.6 \pm 14.5$ years) with chronic (>6 months) hemiparetic gait post-stroke underwent bilateral vastus lateralis muscle biopsies. Methylation status of genomic DNA was assessed by Illumina $450 \mathrm{~K}$ Methylation array. Results We identified 148 ( $p<0.005, \Delta \beta>5 \%)$ CpG loci showing differential DNA methylation status between paretic and nonparetic muscle. Methylation sites mapping to genes related to neuromuscular disorders (12 genes) were over-represented ( $p$ 0.0096). Pathway analysis of the genes regulated by DNA methylation in these loci revealed enrichment for those related to Immune system regulation, connective tissue development including myofibroblast differentiation, nervous system development/function, tissue morphology, mitochondrial functions. Conclusions We report that hemiparetic stroke produces a distinct profile of genomic DNA methylation alterations in paretic leg skeletal muscle that is consistent with phenotypic abnormalities related to energy metabolism, contractile activity, atrophy and structural remodeling. Further understanding of epigenomic mechanisms regulating skeletal muscle structure and function will facilitate the discovery of therapeutic targets for post-stroke rehabilitation and recovery.

Contact Email: hxu@som.umaryland.edu

Disclosure and Study Support: Supported by NIH grants (R01, AG030075, P30 AG028747). 


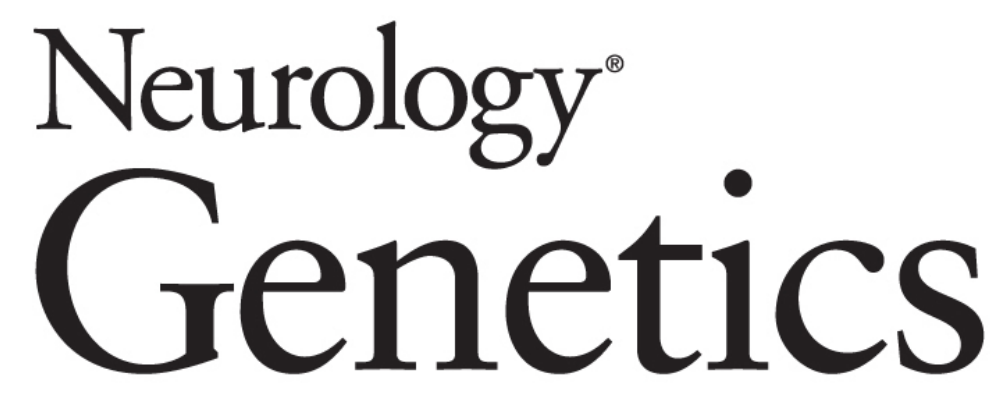

\section{Proceedings of the 21st International Stroke Genetics Consortium Workshop M. Fornage \\ Neurol Genet 2018;4; \\ DOI 10.1212/NXG.0000000000000233}

This information is current as of May 1, 2018

\section{Updated Information \&} Services

Permissions \& Licensing

\section{Reprints}

including high resolution figures, can be found at: http://ng.neurology.org/content/4/2_Supplement_1/e233.full.html

Information about reproducing this article in parts (figures,tables) or in its entirety can be found online at:

http://ng.neurology.org/misc/about.xhtml\#permissions

Information about ordering reprints can be found online: http://ng.neurology.org/misc/addir.xhtml\#reprintsus

Neurol Genet is an official journal of the American Academy of Neurology. Published since April 2015, it is an open-access, online-only, continuous publication journal. Copyright (C) 2018 American Academy of Neurology. All rights reserved. Online ISSN: 2376-7839.

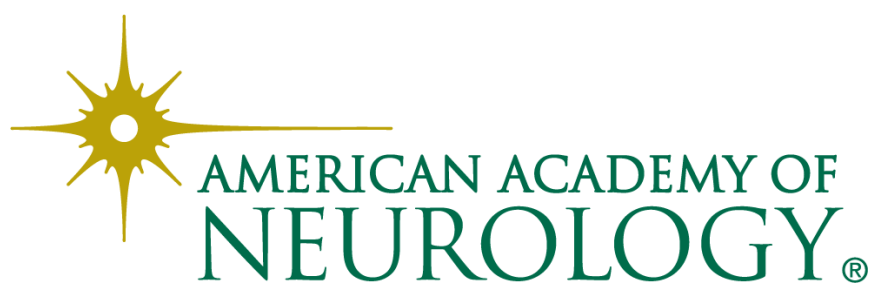

